\title{
Reflection Phase Characterization of Wideband Semi-Periodic Circular High Impedance Surface
}

\author{
Mikal Askarian Amiri and Constantine A. Balanis ${ }^{\star}$, Craig R. Birtcher \\ School of Electrical, Computer and Energy Engineering \\ Arizona State University \\ Tempe, Arizona 85287-5706 USA
}

\begin{abstract}
A wideband semi-periodic circular high impedance surface for loop antenna applications is designed. The surface is illuminated with a plane wavw whose electric field is of phi component. A loop antenna is placed above the surface afterwards to find the operational bandwidth of the structure.
\end{abstract}

\section{INTRODUCTION}

High Impedance Surfaces have been extensively investigated during the past years after they were proposed by Sievenpiper [1] in 1999 and because of their AMC features they were efficiently utilized as antenna ground planes and antenna miniaturization [2], [5]; different geometries of the patches have also been studied. In [4], a transmission line model is proposed for rectangular high impedances surfaces where the period of the structure is much smaller than the wavelength and also the patch size is much bigger than the gap size. In [6], the EBG characteristics of circular strips on a rectangular substrate was investigated and it was shown that an EBG surface with circular symmetry can suppress the surface waves evenly in all directions. [7] was a study of curved high impedance surfaces where the authors characterize the reflection phase of cylindrical structures. In [8], the circular HIS was illuminated with a a plane wave which was of $\phi$ component. Their geometry was then approximated with a rectangular high impedance surface and some design guidelines were proposed afterwards. In [3], reflection phase charateristics of a circularly symmetric high impedance surface under plane wave illumination in cartesian coordinate system was studied.

In this paper, a new type of circular HIS is proposed and since it is going to be used as a ground plane for loop elements, the surface will be illuminated with a TEM wave which is of $\phi$ component. In the last part of the paper the a loop antenna will be placed at a very small height above the HIS to obtain the operational bandwidth of the proposed surface.

\section{Design Geometry and Simulation Results}

Design considered in this paper is based on a Rogers RT/Duroid-5880 disc of thickness $0.635 \mathrm{~cm}$ whose bottom surface is covered with a copper sheet. The top surface of the dielectric is covered by circular rings that have periodic gaps in the angular direction. To start lets illuminate a surface that has strips of thickness $1.35 \mathrm{~cm}$. The distance between the gaps in the radial direction is $0.5 \mathrm{~cm}$. The gap size between the patches on the same ring is 0.1 radians, however distance between the patches on each ring would be different from the other ring. The geometry of the circular HIS is illustrated in Fig. 1. To obtain the reflection phase of the surface it is required to generate a TEM wave whose electric field is of $\phi$ component. This can be done in HFSS by applying PMC(Perfect Magnetic Conductor) to the walls of a coaxial cable. In our case we will introduce a small hole in the middle of our HIS surface and set the radius of the outer conductor of the coaxial cable equal to that of our substrate. This is shown in Fig. 2. The reflection phase of the circular HIS can be seen in Fig. 3 .

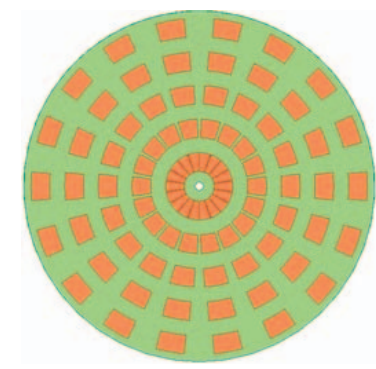

Fig. 1. Top view of the circular HIS

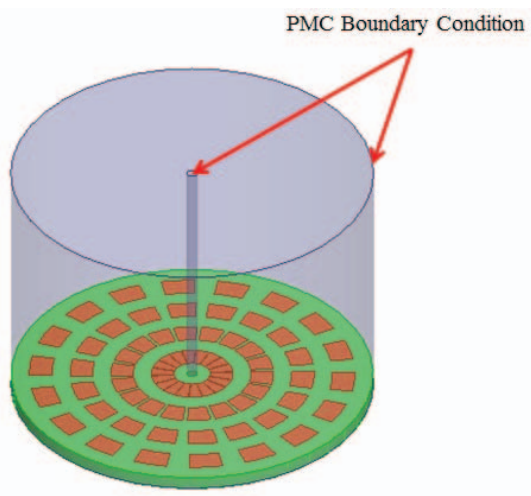

Fig. 2. How to illuminate the surface with circular TEM wave.

As it can be seen in Fig. 3, various modes exist at different frequency intervals. It is believed that each of the rings are having different resonant frequencies. To prove that different rings are responsible for different modes, we can simulate and obtain the reflection phase of each ring separately. Fig. 4 shows how separate rings were simulated. As it can be seen in Fig. 5 each ring resonates at different frequency. Comparing Fig. 3 
and Fig. 5, it is obvious that each mode can be attributed to one the rings. There are still other modes present in the reflection phase of the geometry of Fig. 1 which can attributed to the cross polarization component which is generated because the curved edges of the patches.

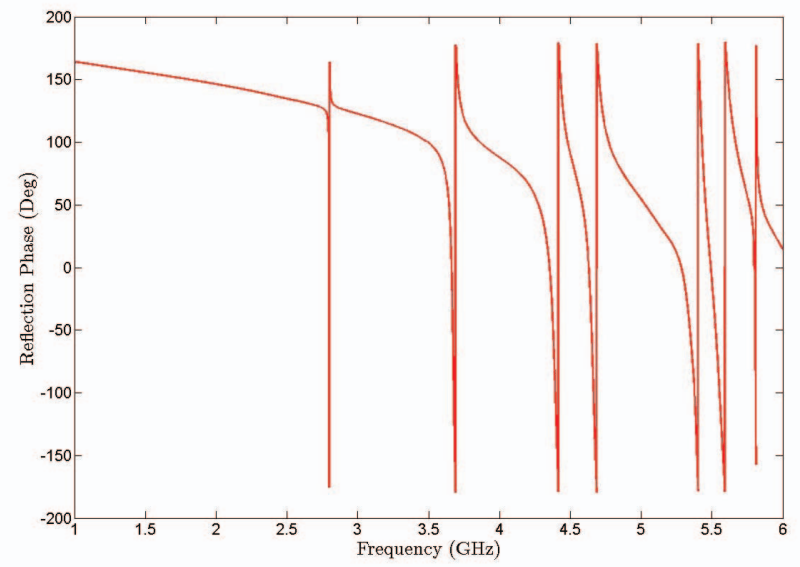

Fig. 3. Reflection phase of the HIS illustrated in Fig. 1

To make the \pm 90 reflection phase bandwidth of the circular HIS wider, we can change the angular gap of each ring so that they resonate at the same frequency. Their reflection phase can be seen in Fig. 6. The modes caused by the cross-polarized component should also shift to higher frequency ranges. This can be controlled by changing the gap size between the adjacent rings. Table I summarizes the geometric parameters of the surface shown in Fig. 7 which was designed to resonate at about $4.2 \mathrm{GHz}$. It is obvious that the angular gaps are the identical on the same ring but they are different on different strips.

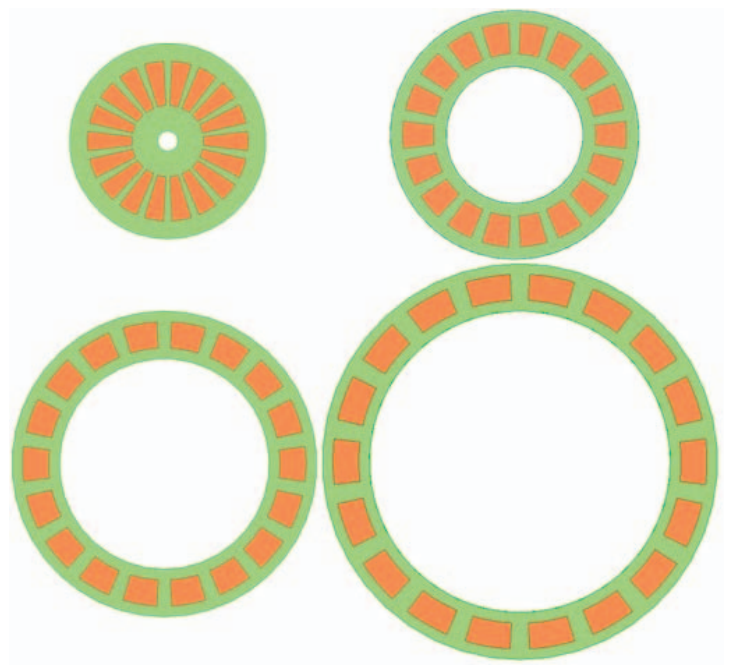

Fig. 4. Separate rings configuration.

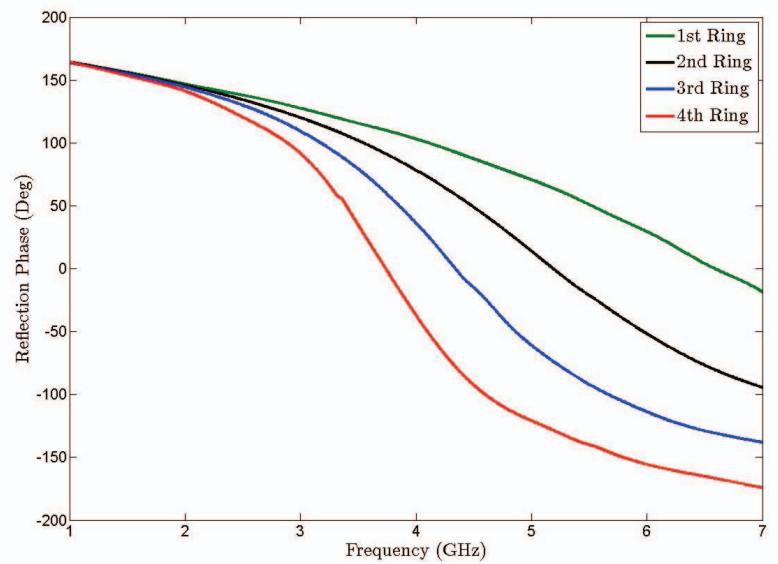

Fig. 5. Reflection phase of the rings shown in Fig. 4.

TABLE I

REFLECTION CHARACTERISTICS OF THE CIRCULAR HIS

\begin{tabular}{|l|l|l|}
\hline Parameter & Angular Gap (deg) & $\begin{array}{l}\text { Thickness } \\
(\mathrm{cm})\end{array}$ \\
\hline $1^{\text {st }}$ ring & 1 & 1.35 \\
\hline $2^{\text {nd }}$ ring & 6.6 & 1.1 \\
\hline $3^{\text {rd }}$ ring & 8.4 & 1.1 \\
\hline $4^{\text {th }}$ ring & 9.5 & 1.2 \\
\hline
\end{tabular}

\section{ANTENNA APPLICATION}

As it is mentioned in [2], to obtain the operational bandwidth of a rectangular HIS, a dipole is placed in the vicinity of it and the dipole length is varied. The frequency interval in which the return loss is below $-10 \mathrm{~dB}$ is the operational bandwidth of the HIS. In our case, since the surface is illuminated with a cylindrical TEM wave, we have to use a circular loop antenna to find the operational bandwidth. Assuming a center frequency of $2.5 \mathrm{GHz}$, the loop is located at $0.01 \lambda$ above the circular HISs and its radius is varied. Fig. 9 demonstrates the side view of the loop element and the

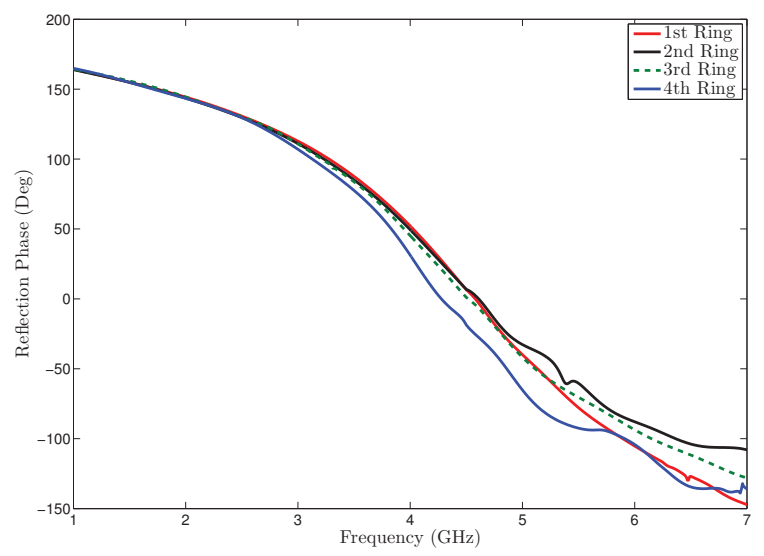

Fig. 6. Reflection phase of the different rings with various angular gaps. 
circular HIS. Figs. 10 shows the return losses for different loop circumferences. The operation bandwidth of the circular HIS is about $24 \%$ at $2.5 \mathrm{GHz}$.

\section{COnClusions}

In this paper a new type of circular HIS surfaces was proposed. It was shown that the angular gaps of the circular strips should be such that all of the rings resonate at the same frequency. The cross-polarized components generated due to the curved edges of the strips can also be moved out from the frequency interval of interest by changing the gaps between the rings. Using this technic the \pm 90 bandwidth of the HIS increased to about $50 \%$ at $4 \mathrm{GHz}$. A loop element was placed at a height of $0.01 \lambda$ above the surface afterwards to find the operational bandwidth of the circular HIS. The operational bandwidth was found to be $24 \%$ at the center frequency of $2.5 \mathrm{GHz}$.

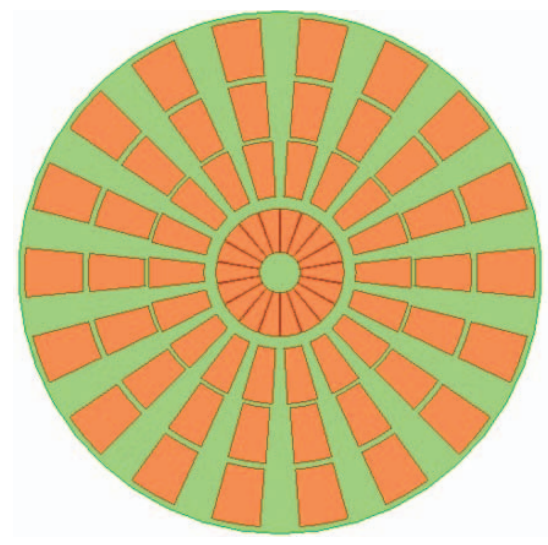

Fig. 7. Geometry of the wideband surface designed for the center frequency of $4.2 \mathrm{GHz}$

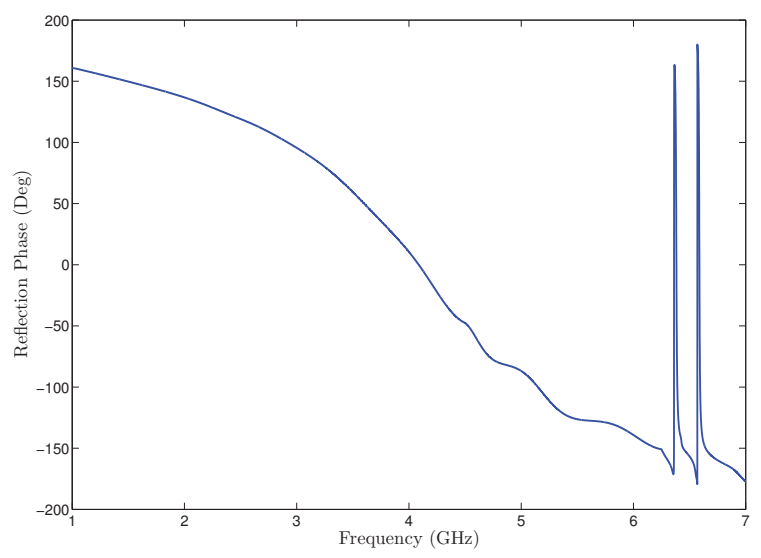

Fig. 8. Reflection Phase of Fig. 7.

\section{REFERENCES}

[1] D. Sievenpiper, "High-Impedance Electromagnetic Surfaces," Ph.D. dissertation, Dept. Elect. Eng., Univ. California, Los Angeles, Los Angeles, CA, USA, 1999.

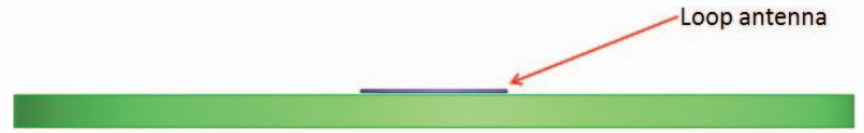

Fig. 9. Side view of the loop antenna at a height of $0.01 \lambda$ above the CHIS.

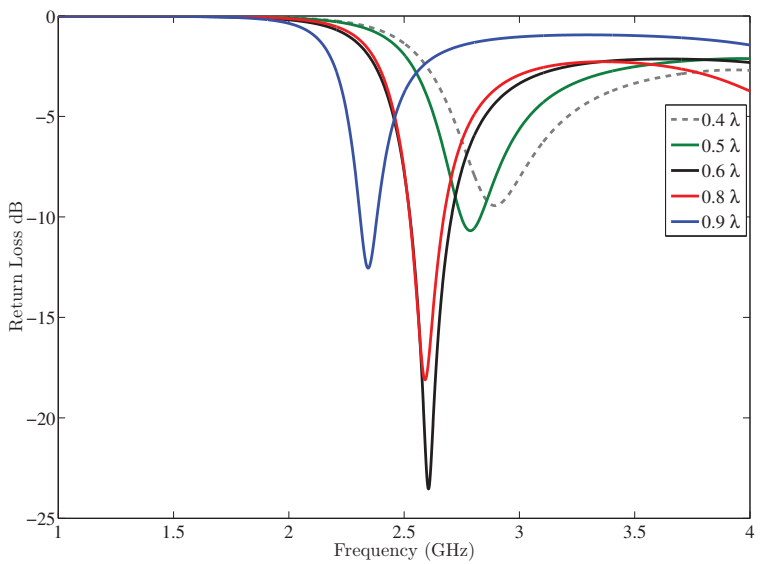

Fig. 10. Return loss of loops with different circumferences.

[2] Yang, Fan and Rahmat-Samii, Yahya, "Reflection phase characterizations of the EBG ground plane for low profile wire antenna applications," IEEE Transactions on Antennas and Propagation, vol. 51, no. 10, pp. 2691 2703, 2003

[3] Mikal. A. Amiri, Constantine A. Balanis, and Ahmet C. Durgun, "Reflection phase characterization of a circularly symmetric high impedance surface,"in Antennas and Propagation Society International Symposium (APSURSI), 2014 IEEE, pp. 1772 - 1773, July 6-11, 2014.

[4] Tretyakov, Sergei., Analytical modeling in applied electromagnetics, Artech House, 2003.

[5] C. A. Balanis, Advanced Engineering Electromagnetics, 2nd ed. New York: Wiley, 2012.

[6] Llombart, Nuria and Neto, Andrea and Gerini, Giampiero and de Maagt, Peter, "Planar circularly symmetric EBG structures for reducing surface waves in printed antennas," IEEE Transactions on Antennas and Propagation, vol. 53, no10., pp. $3210-3218,2005$.

[7] A. C. Durgun, Constantine A. Balanis, Craig R. Birtcher, "Reflection Phase Characterization of Curved High Impedance Surfaces," IEEE Transactions on Antennas and Propagation, vol. 61, no12. , pp. 6030 - 6038, 2013.

[8] Sarrazin, Julien and Lepage, A and Begaud, Xavier, "Circular HighImpedance Surfaces Characterization," IEEE Letters on Antennas and Wireless Propagation, vol. 11, pp. 260 - 263, 2012. 